\title{
Functional hand development in tetraplegia
}

\author{
U Doll, B Maurer-Burkhard, B Spahn and B Fromm \\ Orthopaedic University Hospital Heidelberg, Department II, Germany
}

\begin{abstract}
The development of the 'functional hand' in tetraplegics has been historically facilitated through specialized hand positioning schemes. However, clinical experience at the University Hospital Heidelberg demonstrates no direct relationship between various hand positioning techniques and the probability of functional hand development. The aim of this study was to document the various methods of hand positioning and the resulting functional outcome. In a multi-center study, the paralysed thumb and finger positioning of 64 tetraplegics and the resultant functional outcome was evaluated with a specially developed survey form. Results indicated that the functional outcome of the tetraplegic hand was highly dependent on the level of the spinal cord injury and only to a minor degree dependent upon the different methods of hand positioning employed. The conclusion drawn is that remaining neurologic function after injury determines the final functional outcome of the tetraplegic hand.
\end{abstract}

Keywords: tetraplegic hand function; hand positioning; neurologic situation; level of lesion; physiotherapeutical measures

\section{Introduction}

Therapeutic strategies which aim at the development of a functional hand in newly injured tetraplegics are a key component of the rehabilitation program. Experience shows that the post rehabilitation independence level of tetraplegics is strongly dependent upon, inter alia, dealing with the remaining neurological and vegetative function of hands and fingers. (Figure 1). ${ }^{1}$

The commonly applied concept used in rehabilitative settings is the use of various hand positioning techniques which supposedly favor the development of a functional hand shape. ${ }^{1,2}$ Clinical experience and observations of this concept in the Rehabilitation Center of the Orthopaedic University Hospital Heidelberg have shown widely varying results. Patients receiving the same type of hand positioning experienced results ranging from well developed to completely insufficient functional hand development.

These varying results led to the following study question: Can the development of a tetraplegic functional hand be influenced by a particular hand positioning scheme?

\section{Subjects and method}

\section{Patients}

The questioning was carried out in 64 patients with complete or incomplete tetraplegia. The average age

Correspondence: U Doll, Orthopaedic University Hospital, Schlierbacher Landstr. 200a, 69118 Heidelberg, Germany was 34.8 years, ranging from $13-73$ years. The classification of the patients $(n=60)$ according to the level of paralysis is shown in Figure 2. Four patients with an incomplete paralysis (tetraparesis) were excluded from the study, because they could not be assigned to a homogenous group. Only patients with a complete paralysis from $\mathrm{C} 2 / 3-\mathrm{C} 7 / 8$ were included in the study.

\section{Exclusion criteria}

Exclusion criteria were:

- additional neurologic diseases, e.g. traumatic brain injury or tumors.

- operations performed at the upper extremity to improve hand function.

- fractures in the hand area.

- children under the age of 12 , because of the inability to answer survey questions.

\section{Positioning group}

Forty-four patients received rehabilitative hand positioning. Eighteen were from the Orthopaedic University Hospital Heidelberg, and 26 from other hospitals. Hands were considered positioned:

1. If hand positioning was begun at the beginning of rehabilitation.

2. If hands were positioned with an aid (e.g. positioning-glove, Figure 3) for at least $12 \mathrm{~h}$ per day or night for 3 months. ${ }^{1,2}$ 


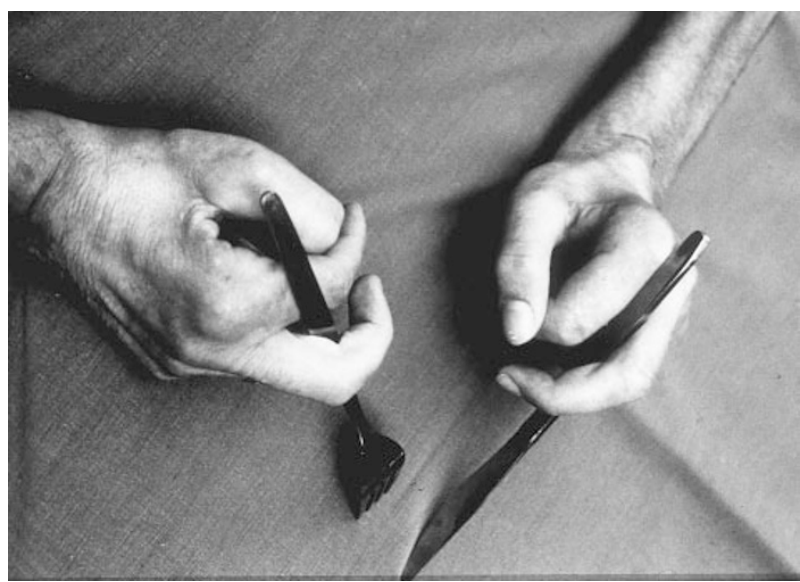

Figure 1 Independence in activities of daily life

\section{Level of lesion $n=64$}

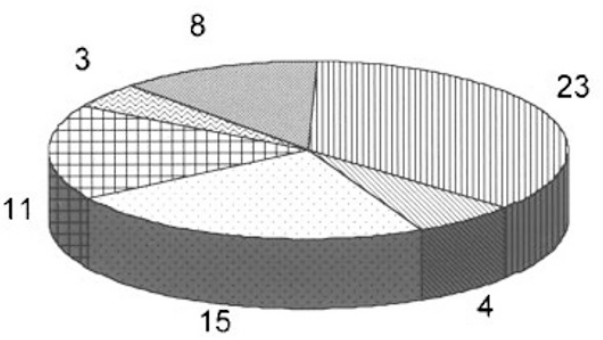

\begin{tabular}{|c|c|c|}
\hline incomplete & $\sqsubset \mathrm{C} 6 / 7$ & 田 C 7/8 \\
\hline
\end{tabular}

Figure 2 Levels of neurologic lesions

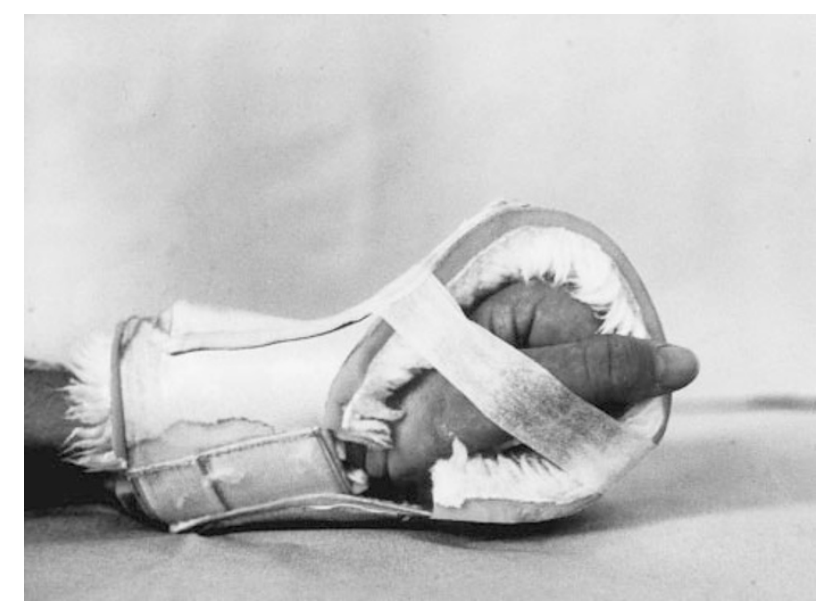

Figure 3 Positioning glove for a tetraplegic hand

\section{Types of positioning}

Classical

Thirty-four patients were positioned in the classical functional hand position. By this we understand dorsal extension of the wrist with flexion of the MP and PIP joints. Of this group, 19 patients were positioned with a positioning glove, ten with palmar rolls, and five patients used a palmar splint for positioning.

Other positioning techniques

Ten patients were positioned in 0 degree wrist extension with fully extended finger joints. Ten patients had a wrist extension splint, and two patients received additionally an arm splint immobilising the elbow joint in an extended position.

Non-positioning group

Sixteen patients obtained no hand positioning.

Survey implementation

The hand positioning technique and the subsequent development of a functional hand for 64 tetraplegics were documented using a survey form. The patients

\author{
Hand Evaluation Scheme \\ Name \\ Date of birth \\ date of injury \\ kind of injury \\ fracture (spine)
}

level of paralysis initially

level of paralysis now

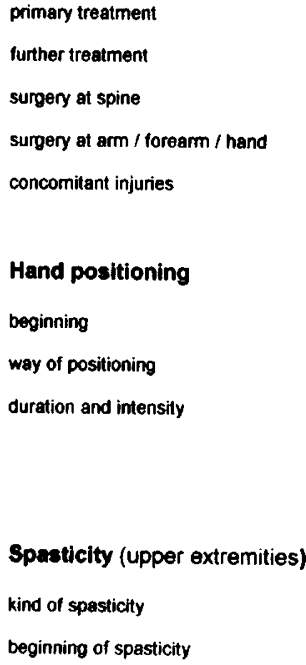

Figure 4 Hand evaluation scheme 
were questioned individually by members of the rehabilitation team (Figures 4 and 5). Additionally the patients hands were photo-documented. At least 6 months after the beginning of the hand positioning program both groups of patients were rated by the clinical team as having a functional or non functional hand. The following classification scheme was used to rate the hands.

\section{Criteria for rating hands as functional hands}

A tetraplegic functional hand (Figure 6) is developed by an active dorsal extension in the wrist due to an innervated extensor carpi radialis muscle. Because of shortened finger flexors, wrist extension results in the passive closing of the fingers. The finger arrangement in the metacarpo phalangeal (MP) and proximal interphalangeal (PIP) joints is then arranged like a flight of stairs, with a maximum flexion of the little finger, decreasing towards the index finger. ${ }^{3,4}$ Furthermore, an active dorsal extension of the wrist causes an opposition of the thumb against the index finger. This allows for the grasping of light objects in a key grip or

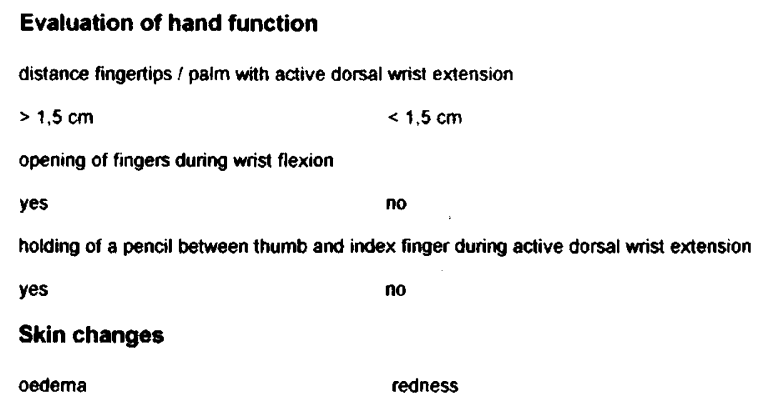

oedema

redness

Pain history

Independence

dorminant arm before injury

dominant arm after injury

Splints / braces

yes

no

kind

Appliances for work

Job

Driving licence

yes

Date

Signature

Figure 5 Hand evaluation scheme lateral grip fashion. A passive palmar flexion of the wrist results in the opening of the hand. Thus, objects can be grasped and released. If a hand did not meet all of these demands it was classified as a "non functional hand'.

\section{Results}

Hand positioning

Of the 44 patients with hand positioning, ten $(23 \%)$ developed a functional hand. (Four of them had their finger joints positioned in full extension and their wrist joint in 0 degree flexion, and six patients were positioned in the classical hand position). Even a hand positioning in 0 degree wrist extension and fully extended fingers, an absolute contradiction to the classical hand positioning, could not prevent the development of a functional hand in $1 / 3$ of all patients. Thirty-four $(77 \%)$ patients developed no functional hand.

\section{No hand positioning}

Of the 16 patients without hand positioning, eight $(50 \%)$ patients developed a functional hand and eight $(50 \%)$ did not.

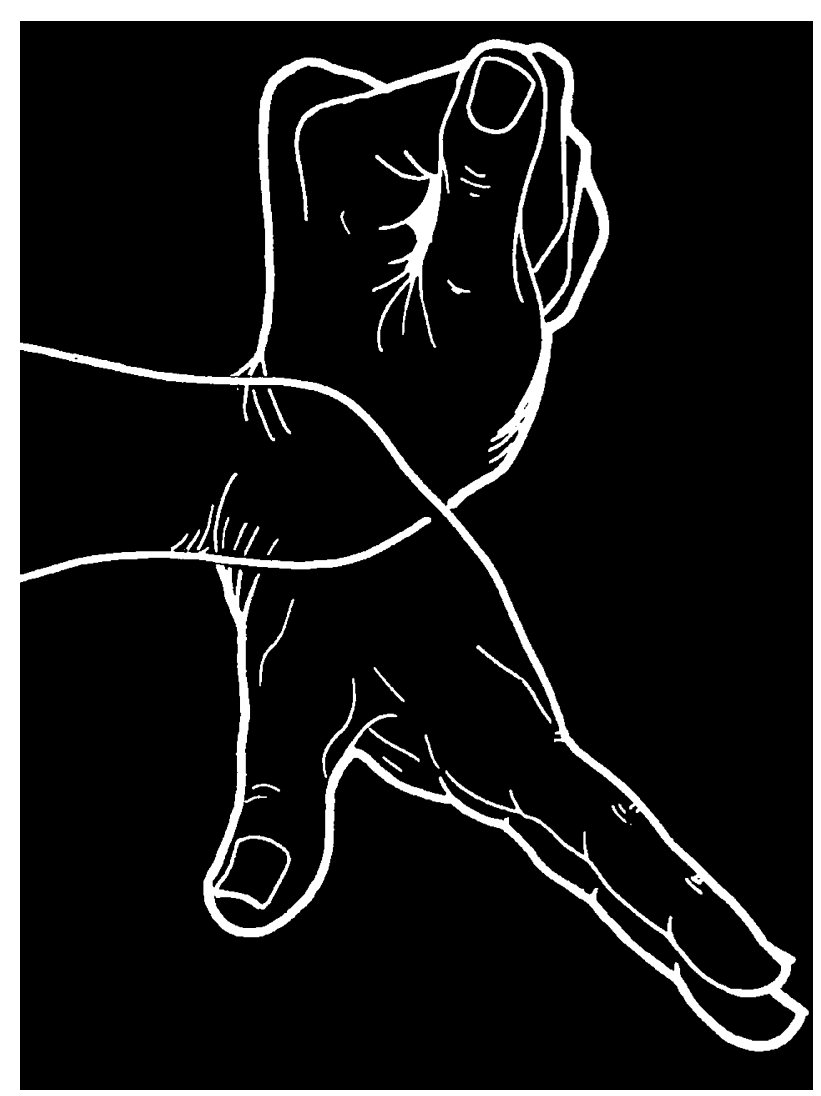

Figure 6 Grasping mechanism of a tetraplegic functional hand 
Table 1 Results of patients without functional hand positioning

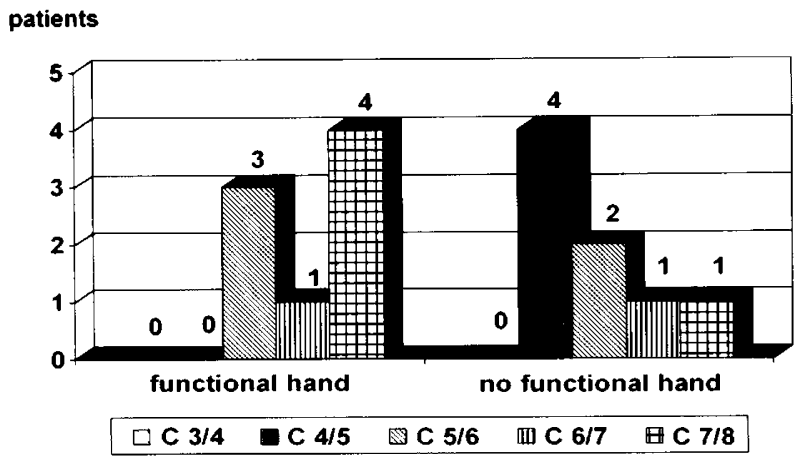

Table 2 Results of patients with functional hand positioning patients

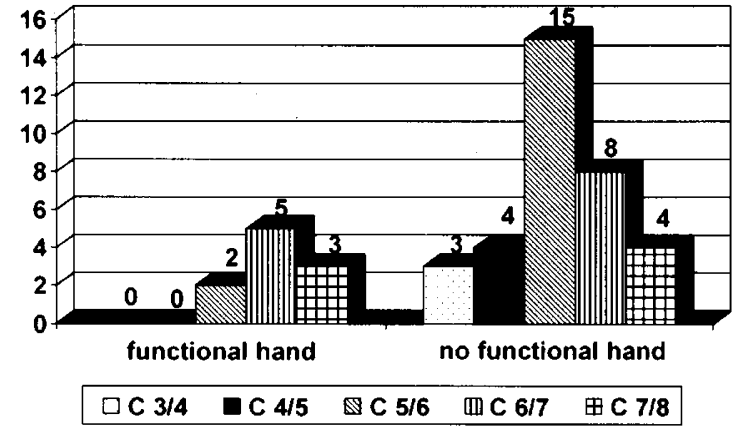

Functional hand development and neurological lesion level

The hands were also classified according to the different neurological lesion levels. Tables 1 and 2 show that the probability of functional hand development is higher with lower lesions.

\section{Discussion}

Our results demonstrate that for the development of a tetraplegic functional hand, particular hand positioning is not absolutely necessary. Furthermore, consistent hand positioning does not necessarily lead to the development of a functional hand. Thus, a tetraplegic hand kept in its natural position with slight dorsal wrist extension and moderate finger flexion (a result of active dorsal extension), and a positioning of the shoulders in slight external rotation would be sufficient for the development of a tetraplegic functional hand without particular hand positioning (Figure 7).

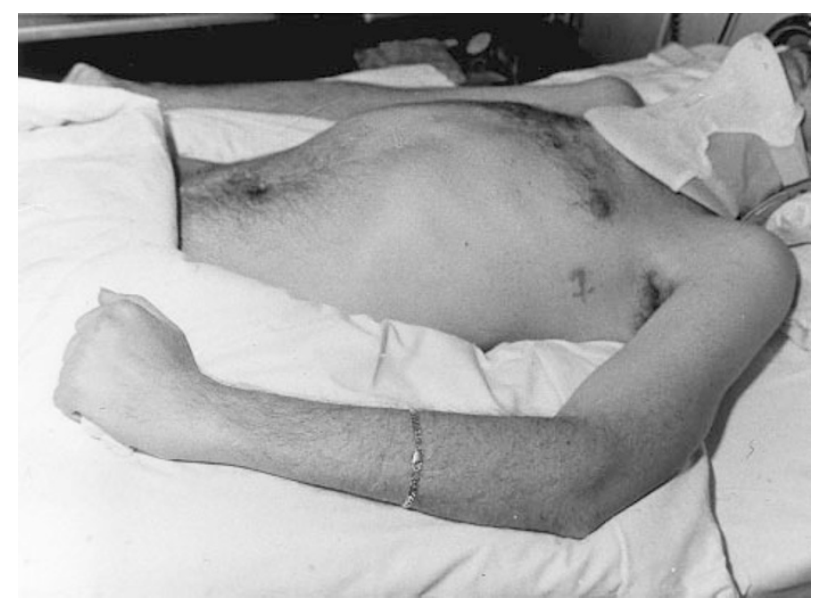

Figure 7 Ideal positioning of a tetraplegic hand

It is our impression that the neurological situation of the patient, the innervation and especially the spasticity determines the functional outcome and quality of movement associated with the tetraplegic functional hand.

A future goal of the clinical team should be to look for other possible rehabilitation schemes which favour the development of a functional hand. In any human being, tetraplegic or other, the development of the hand as a supporting and grasping organ is dependent on the upright mechanism of the whole body. In the case of neurological lesions, therapists should attempt to achieve the best possible coordinated movement of the muscles by neurophysiologically based therapies such as Vojta, PNF, or Bobath.

In order to maximize this functional ability, the anatomical and functional condition of the normally innervated hand should be taken into consideration as well as muscle tone and positioning of the whole body.

\section{Acknowledgements}

Thanks to Mr E Maurer and Mr Walter Accles for their assistance in translating this study.

\section{References}

1 Paeslack V, Schlüter H. Physiotherapie in der Rehabilitation Querschnittgelähmter. Springer Verlag Berlin-Heidelberg-New York 1980, pp 22-25.

2 Gerner J. Die Querschnittlähmung. Blackwell Wissenschaft Berlin 1992, pp 45-49.

3 Rauber, Kopsch, Hrsg, Leonhardt H, Töndury G, Tillmann B, Zilles K. Anatomie des Menschen Bewegungsapparat. Thieme Verlag Stuttgart-New York 1987, pp 405-442.

4 Kapandji. Funktionelle Anatomie der Gelenke, obere Extremität. Enke-Verlag Stuttgart 1984, pp 164-280. 